Evaluation of an automated assay based on monoclonal anti-human serum amyloid $A$ (SAA) antibodies for measurement of canine, feline and equine SAA

Christensen, Mette; Jacobsen, Stine; Ichiyanagi, T.; Kjelgaard-Hansen, Mads

Published in:

The Veterinary Journal

DOI:

10.1016/j.tvjl.2012.05.007

Publication date:

2012

Document version

Early version, also known as pre-print

Citation for published version (APA):

Christensen, M., Jacobsen, S., Ichiyanagi, T., \& Kjelgaard-Hansen, M. (2012). Evaluation of an automated assay based on monoclonal anti-human serum amyloid A (SAA) antibodies for measurement of canine, feline and equine SAA. The Veterinary Journal, 194(3), 332-337. https://doi.org/10.1016/j.tvjl.2012.05.007 


\title{
Evaluation of an automated assay based on monoclonal anti-human serum amyloid A (SAA) antibodies for measurement of canine, feline, and equine SAA is
}

\author{
M. Christensen ${ }^{\mathrm{a}, *}$, S. Jacobsen ${ }^{\mathrm{b}}$, T. Ichiyanagi ${ }^{\mathrm{c}}$, M. Kjelgaard-Hansen ${ }^{\mathrm{a}}$ \\ ${ }^{a}$ Department of Small Animal Clinical Sciences, University of Copenhagen, Denmark \\ ${ }^{\mathrm{b}}$ Department of Large Animal Sciences, University of Copenhagen, Denmark \\ ' EIKEN Chemical Company, Tokyo, Japan
}

\section{A R T I C L E I N F O}

\section{Article history:}

Accepted 7 May 2012

\section{Keywords:}

Immunoassay

Validation

Acute phase protein

Companion animals

Amyloid

\begin{abstract}
A B S T R A C T
Major acute phase proteins (APPs) have proven diagnostically useful in dogs, cats and horses with routine use facilitated by commercially available automated heterologous assays. An automated assay applicable across all three species would highly facilitate further dissemination of routine use, and the aim of this study was to validate an automated latex agglutination turbidimetric immunoassay based on monoclonal anti-human serum amyloid A (SAA) antibodies for measurement of canine, feline and equine SAA. Serum samples from 60 dogs, 40 cats and 40 horses were included. Intra- and inter-assay imprecision, linearity and detection limit (DL) were determined to assess analytical performance. To assess clinical performance, equine and feline SAA measurements were compared with parallel measurements using a previously validated automated SAA assay in a method comparison setting, and by assessing overlap performance of canine SAA in healthy dogs and diseased dogs with and without systemic inflammation.

Intra- and inter-assay CVs ranged between $1.9-4.6 \%$ and between $3.0-14.5 \%$, respectively. Acceptable linearity within a clinically relevant range of SAA concentrations was observed for all three species. The DL was $1.06 \mathrm{mg} / \mathrm{L}$. Method comparison revealed acceptable agreement of the two assays measuring feline and equine SAA, and the overlap performance of canine SAA was acceptable. The tested assay measured SAA in canine, feline and equine serum with analytical and overlap performance acceptable for clinical purposes so improving practical aspects of clinical APP application. The monoclonal nature of the antibodies suggests strong, long-term inter-batch performance stability.
\end{abstract}

(c) 2012 Elsevier Ltd. All rights reserved.

\section{Introduction}

Major acute phase proteins (APPs) have proven to be diagnostically useful as routine inflammatory markers in major companion animal species including dogs, cats, and horses (Sasaki et al., 2003; Jacobsen and Andersen, 2007; Nakamura et al., 2008). Automated human turbidimetric immunoassays (TIA) have previously been validated for measurements of feline and equine serum amyloid A (SAA) (Hansen et al., 2006; Jacobsen et al., 2006a) and canine C-reactive protein (CRP) (Kjelgaard-Hansen et al., 2003; Kjelgaard-Hansen, 2010; Klenner et al., 2010), facilitating dissemination of the routine applicability of APPs (Kjelgaard-Hansen and Jacobsen, 2011). However, even though their diagnostic potential has been known for decades, routine measurement of APPs is still not as widely applied as expected (Eckersall, 2004).

\footnotetext{
Part of this study was presented at the 13th Conference of the European Society of Veterinary Clinical Pathology, Dublin, 3 September 2011.

* Corresponding author. Tel.: +45 35332952.

E-mail address: mc@life.ku.dk (M. Christensen).
}

Development of an automated assay applicable for measurements of major APPs across all three species (canine, feline and equine) would markedly improve the practical aspects and dissemination of measuring major APPs for routine diagnostic purposes. SAA is an obvious target for a multi-species assay as it is a major APP in all species of interest in veterinary medicine (KjelgaardHansen and Jacobsen, 2011). Moreover, the diagnostic potential of SAA measurement in feline and equine medicine is well established (Sasaki et al., 2003; Jacobsen and Andersen, 2007) and several studies have shown that canine SAA also can be diagnostically useful as a routine inflammatory marker most likely comparable to the diagnostic capacity of other canine APPs (Chikamune et al., 1998; Dabrowski et al., 2007, 2009). For diagnostic laboratories investing in APP assays, the presence of a single assay for several animal species would be an obvious advantage (Kjelgaard-Hansen and Jacobsen, 2011). Furthermore, if the assay was based on monoclonal antibodies, stable long-term and inter-batch performance would be possible (Kjelgaard-Hansen, 2010).

The aim of the present study was to evaluate the analytical performance of an automated latex agglutination turbidimetric immunoassay (LAT) based on monoclonal anti-human SAA 
antibodies to measure SAA in dogs, cats, and horses. The evaluation included an assessment of imprecision, inaccuracy and detection limit (DL), as well as a comparison with an established method for diagnostic measurements of feline and equine SAA and an evaluation of the overlap performance of canine SAA.

\section{Materials and methods}

Assay

An LAT based on monoclonal anti-human SAA antibodies was evaluated in the study. The assay was developed in collaboration with EIKEN Chemical Company for measurements of canine, feline, and equine SAA. Data demonstrating the specificity of the monoclonal antibodies in the detection of SAA are provided in Appendix A (Supplementary material). The measurements with the LAT and the commercial available human SAA TIA (Hansen et al., 2006; Jacobsen et al., 2006a) were performed using an automated clinical chemistry analyser (ADVIA 1800, Siemens). The LAT was calibrated using heterologous calibration material, meaning that the concentrations measured in $\mathrm{mg} / \mathrm{L}$ were in human equivalents of SAA, rather than exact concentrations of species-specific SAA.

\section{Samples}

Serum samples from 60 dogs, 40 cats and 40 horses obtained for diagnostic purposes were included in the study. All samples were taken from client-owned animals. The study was approved by the local ethical committee. All animals underwent clinical examination during the period January 2010 to May 2011, and blood samples were analysed at the Central Laboratory, Department of Small Animal Clinical Sciences, University of Copenhagen, Denmark. Remaining serum was stored in plastic vials at $-20^{\circ} \mathrm{C}$ until analysis. Samples were only thawed when needed for analysis in order to limit freeze-thaw cycles.

\section{Assay characteristics}

The intra- and inter-assay variations were determined as the coefficient of variation (CV) based on replicate measurements of SAA in serum pools within the same analytical run (same day) ( $n=8-16)$ and across analytical runs (different days) $(n=6-8)$, respectively. Pools containing intermediate concentrations of SAA (40$45 \mathrm{mg} / \mathrm{L}$ for horse and cat and $70-75 \mathrm{mg} / \mathrm{L}$ for dogs, respectively) and pools with high concentrations of SAA ( $>900 \mathrm{mg} / \mathrm{L}$ for canine SAA, $>170 \mathrm{mg} / \mathrm{L}$ for feline SAA, and $>1500 \mathrm{mg} / \mathrm{L}$ for equine SAA) were used. The DL was calculated from replicate determinations of blanks (distilled water) $(n=8)$.

Inaccuracy was investigated by linearity under dilution. Pooled samples with very high concentrations of canine and feline SAA were initially diluted $0 \%, 10 \%$, $20 \%, 30 \%, 40 \%, 50 \%, 60 \%, 70 \%, 80 \%, 90 \%$ and $100 \%$ using distilled water; subsequently, in order to get a more detailed picture of the inaccuracy at high and low concentrations, additional dilutions were made (5\%,15\% and 95\%). Because extremely high concentrations of SAA are occasionally observed in equine serum (Jacobsen et al., 2006b), an even more detailed spectrum of dilutions of such an extreme sample were made for this particular species, namely, $0 \%, 1 \%, 2 \%, 3 \%, 4 \%, 5 \%, 6 \%, 7 \%$, $8 \%, 9 \%, 10 \%, 15 \%, 20 \%, 25 \%, 30 \%, 35 \%, 40 \%, 45 \%, 50 \%, 55 \%, 60 \%, 65 \%, 70 \%, 75 \%, 80 \%$, $85 \%, 90 \%, 95 \%$ and $100 \%$. This was done to test for possible pro-zone effect having a clinical impact. Expected concentrations of SAA for each dilution were calculated from the observed concentrations in undiluted canine and feline serum and in $25 \%$ dilution of the equine serum, respectively, and the linearity was investigated visually and by linear regression.

A method comparison for measurements of feline and equine SAA was performed comparing results obtained by the LAT to measurements of SAA using a commercial available TIA validated for SAA measurements in cats and horses (Hansen et al., 2006; Jacobsen et al., 2006a). Parallel measurements of SAA in 40 individual equine serum samples and 40 individual feline serum samples were used for the method comparisons (Jensen and Kjelgaard-Hansen, 2006). The serum samples were chosen in order to cover the full spectrum of SAA concentrations most frequently expected in clinical settings. The LAT was considered reliable for diagnostic measurements of feline and equine SAA if the performance could be shown to be comparable to the reference method already used for routine diagnostic measurements of SAA (Jacobsen and Andersen, 2007).

\section{Overlap performance}

SAA is a major positive APP in dogs (Cerón et al., 2005). If the LAT should be considered reliable for diagnostic assessment of the acute phase response, the overlap performance of SAA measured by the LAT should be acceptable and able to discriminate dogs with inflammatory disease from healthy dogs and dogs without inflammatory disease (Kjelgaard-Hansen et al., 2003; Nakamura et al., 2008; Yuki et al., 2010; Hagman, 2011). Based on the final clinical diagnoses, 60 dogs were retrospectively assigned to three groups: (1) clinically healthy dogs $(n=20)$; (2) dogs with systemic inflammation $(n=20)$, and (3) diseased dogs without systemic inflamma- tion $(n=20)$. For all dogs basic hematologic and clinical chemical profiles were analysed (Jensen et al., 2001). Additional diagnostic tests for each dog were conducted at the discretion of the individual clinician (e.g. radiography, ultrasonography, MRI, CSF, endocrine testing, cytology or histopathology).

Clinically healthy dogs had unremarkable findings on physical and clinical pathological examinations $(n=20)$. Diseased dogs without systemic inflammation were diagnosed with idiopathic epilepsy $(n=7)$, syringomyelia $(n=2)$, cardiac disorders (myxomatous mitral valve disease or dilated cardiomyopathy) $(n=3)$, arthrosis $(n=2)$ or other disorders without any signs of systemic inflammation $(n=6)$. Dogs classified with systemic inflammation were hospitalized because of trauma $(n=4)$, pyometra $(n=2)$, severe meningitis $(n=3)$, acute gastroenteritis $(n=4)$, acute symptoms of chronic gastrointestinal disorders $(n=2)$ or other disorders with a systemic inflammatory response $(n=5)$.

A comparable spectrum of ages, sexes, and breeds were included in each group.

\section{Statistics}

Intra- and inter-assay CVs were calculated using routine descriptive statistical procedures (Büttner et al., 1980). Imprecision performance was deemed acceptable if comparable to previously validated automated assays measuring major APPs, which have demonstrated sufficient analytical performance for clinical applicability (Kjelgaard-Hansen et al., 2003; Hansen et al., 2006; Jacobsen et al., 2006a). Investigation of linearity under dilution was accomplished by Deeming regression analysis. Linearity performance was deemed acceptable if slope and Y-intercept did not deviate from 1 and 0 , respectively.

The method comparison was performed by Deeming regression, and the Pearson's correlation coefficients were calculated. Agreement between methods was assessed by deviations from line of agreement $(\mathrm{Y}=\mathrm{X})$. Pearson's correlation coefficients close to 1 indicated positive correlation.

Runs test was performed in all types of regression analyses to determine whether data deviated significantly from the applied linear model. Overlap performance was assessed by comparison of SAA concentrations among groups of dogs using Dunn's multiple comparison test and visual assessment of scatter plots. Significant discrimination between the group of dogs with systemic inflammation and other groups were set as criteria for acceptability. Significance was set at $P<0.05$.

\section{Results}

\section{Assay characteristics}

Intra- and inter-assay CVs ranged from $1.9 \%$ to $4.6 \%$ and from $3.0 \%$ to $14.5 \%$, respectively, in measurements of high and intermediate concentrations of SAA (Table 1 ). When investigating linearity under dilution, a deviation from linearity was observed in the measurements of high concentrations of SAA limiting the working range of the assay for all three species (Fig. 1A-C). Introduction

\section{Table 1}

Intra- and inter-assay coefficients of variation (CV) for canine, feline, and equine serum amyloid A (SAA) measured by a latex agglutination immunoassay based on monoclonal anti-human SAA antibodies. SD, standard deviation.

\begin{tabular}{|c|c|c|c|}
\hline & Canine SAA & Feline SAA & Equine SAA \\
\hline \multicolumn{4}{|c|}{ Intra-assay CV Intermediate concentration } \\
\hline Range (mg/L) & {$[67.7 ; 75]$} & {$[39.9 ; 46.2]$} & {$[42.8 ; 45.2]$} \\
\hline Mean $(\mathrm{mg} / \mathrm{L})$ & 72.6 & 44.1 & 43.5 \\
\hline SD & 3.0 & 2.0 & 0.9 \\
\hline CV (\%) & 4.1 & 4.6 & 2.1 \\
\hline \multicolumn{4}{|c|}{ Intra-assay CV High concentration } \\
\hline Range (mg/L) & {$[909.2 ; 973.6]$} & {$[170.5 ; 188.9]$} & {$[1491 ; 1668]$} \\
\hline Mean $(\mathrm{mg} / \mathrm{L})$ & 931.6 & 177.6 & 1612 \\
\hline SD & 17.5 & 7.1 & 52.1 \\
\hline CV (\%) & 1.9 & 4.0 & 3.2 \\
\hline \multicolumn{4}{|c|}{ Inter-assay CV Intermediate concentration } \\
\hline Range (mg/L) & {$[55.2 ; 77.5]$} & {$[39.4 ; 46.1]$} & {$[34.3 ; 46.1]$} \\
\hline Mean $(\mathrm{mg} / \mathrm{L})$ & 68.6 & 42.7 & 41.3 \\
\hline SD & 8.6 & 2.9 & 4.8 \\
\hline CV (\%) & $12.5 \%$ & $6.7 \%$ & 11.6 \\
\hline \multicolumn{4}{|c|}{ Inter-assay CV High concentration } \\
\hline Range (mg/L) & {$[687.5 ; 946.0]$} & {$[130.5 ; 188.9]$} & {$[1491 ; 1668]$} \\
\hline Mean (mg/L) & 801.5 & 160.8 & 1543 \\
\hline SD & 77.6 & 23.3 & 46.0 \\
\hline CV (\%) & 9.7 & 14.5 & 3.0 \\
\hline
\end{tabular}



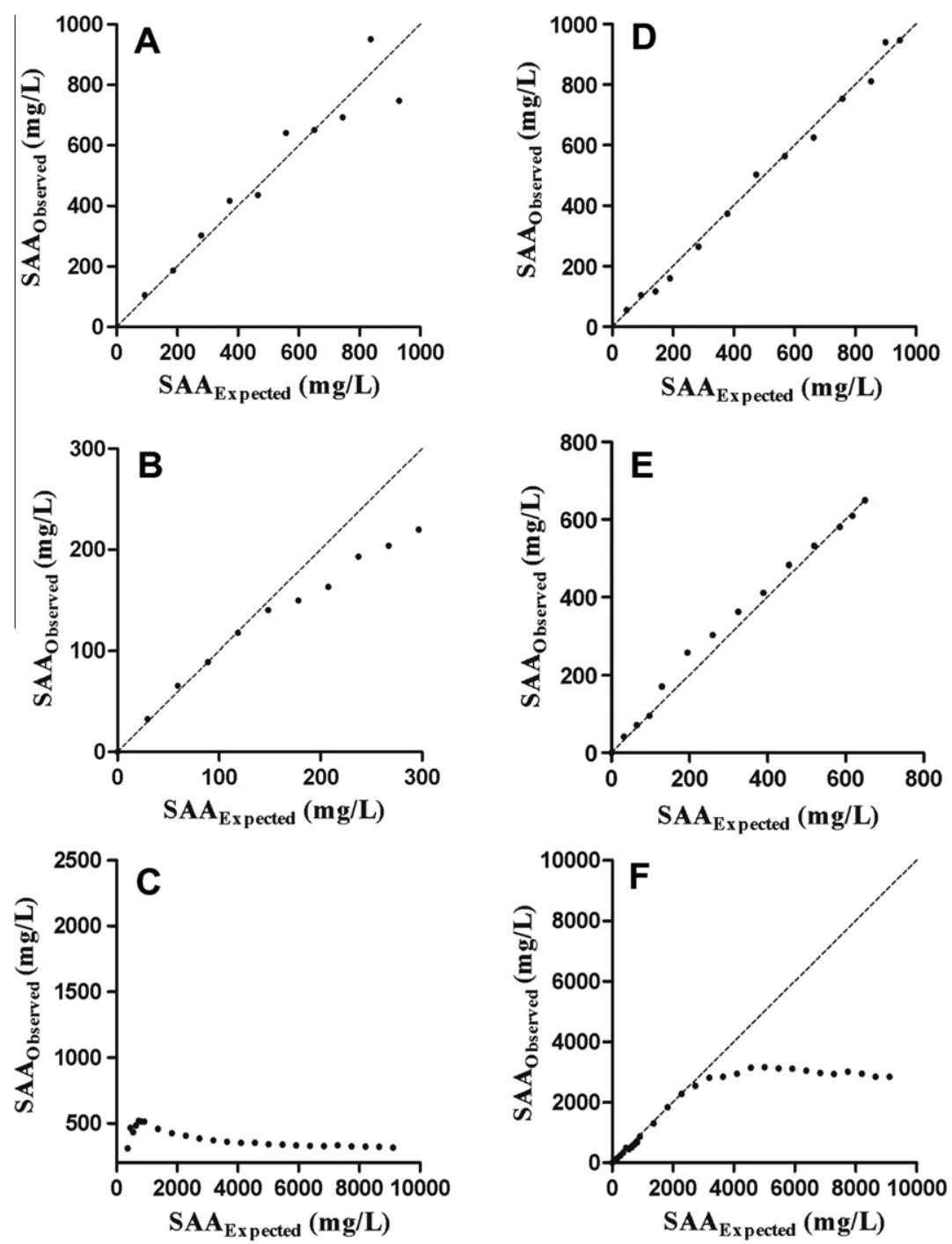

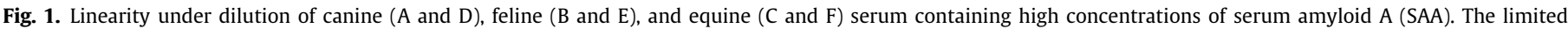

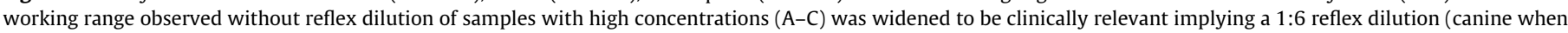
$>300 \mathrm{mg} / \mathrm{L}$; equine when $>300 \mathrm{mg} / \mathrm{L}$, and feline when $>100 \mathrm{mg} / \mathrm{L})(\mathrm{D}-\mathrm{F})$. The dashed lines illustrate lines of agreement $(\mathrm{Y}=\mathrm{X})$.

of a 1:6 reflex dilution of canine and equine samples with SAA concentrations $>300 \mathrm{mg} / \mathrm{L}$ and feline samples with SAA concentrations $>100 \mathrm{mg} / \mathrm{L}$ expanded the range of acceptable linearity to a clinically relevant range for all species investigated (Fig. 1D-F). After reflex dilution, acceptable linearity was observed in the following working range: $0-1000 \mathrm{mg} / \mathrm{L}$ for canine SAA, $0-700 \mathrm{mg} / \mathrm{L}$ for feline SAA and $0-2500 \mathrm{mg} / \mathrm{L}$ for equine SAA (Fig. 1D-F) with observed

\section{Table 2}

Linearity under dilution of canine, feline and equine serum pools with high serum amyloid A (SAA) concentrations after reflex dilution of canine and equine samples with SAA concentrations exceeding $300 \mathrm{mg} / \mathrm{L}$ and feline samples containing SAA concentrations above $100 \mathrm{mg} / \mathrm{L}$. $\mathrm{CI}$, confidence interval; $P$ (runs test), probability for fitting a linear model.

\begin{tabular}{llll}
\hline & Canine SAA & Feline SAA & Equine SAA $(<2500 \mathrm{mg} / \mathrm{L})$ \\
\hline Y-intercept & 4.03 & 15.0 & -47.7 \\
{$[95 \% \mathrm{CI}]$} & {$[-22.95 ; 31.0]$} & {$[-1.3 ; 31.3]$} & {$[-104 ; 8.9]$} \\
Slope & 1.0 & 1.03 & 1.034 \\
{$[95 \% \mathrm{CI}]$} & {$[0.95 ; 1.05]$} & {$[0.98 ; 1.07]$} & {$[0.98 ; 1.09]$} \\
$P$ (Runs test) & 0.51 & 0.025 & 0.226 \\
$r$ & 0.994 & 0.997 & 0.996 \\
\hline
\end{tabular}

slopes and Y-intercept not deviating from 1 and 0 , respectively (Table 2). Specifically for equine samples the quantitative ability ceased above $2500 \mathrm{mg} / \mathrm{L}$, however, importantly no clinically relevant prozone effect for concentrations up to $10000 \mathrm{mg} / \mathrm{L}$ were observed, meaning that no tendencies of false low results were seen, even with significant excess of antigen in the antigen-antibody reactions of the assay. DL was estimated to be $1.06 \mathrm{mg} / \mathrm{L}$.

\section{Method comparison}

A high degree of correlation was observed between SAA concentrations measured by LAT and measurements using the previously validated commercially available TIA (Table 3). Method comparison revealed a proportional disagreement for measurements of both feline and equine SAA (slope differing from 1) and an additional systematic inaccuracy for equine SAA (Y-intercept differing from 0) (Table 3). The proportional disagreement was confirmed by direct plot of SAA measurements obtained by the two assays (Fig. 2A and B), visualising the tendency of the LAT to measure higher concentrations of SAA than the previously validated TIA. 
Table 3

Deeming regression of the method comparison between equine and feline serum amyloid A (SAA) measured by the novel latex agglutination turbidimetric immunoassay and a previously validated commercial available method.

\begin{tabular}{lll}
\hline & Feline SAA & Equine SAA \\
\hline Slope & 4.7 & 1.34 \\
{$[95 \% \mathrm{CI}]$} & {$[4.11 ; 5.32]$} & {$[1.17 ; 1.5]$} \\
Y-intercept & -24.5 & 275 \\
{$[95 \% \mathrm{CI}]$} & {$[-56.0 ; 7.07]$} & {$[98.7 ; 452]$} \\
$r$ & 0.87 & 0.87 \\
\hline
\end{tabular}
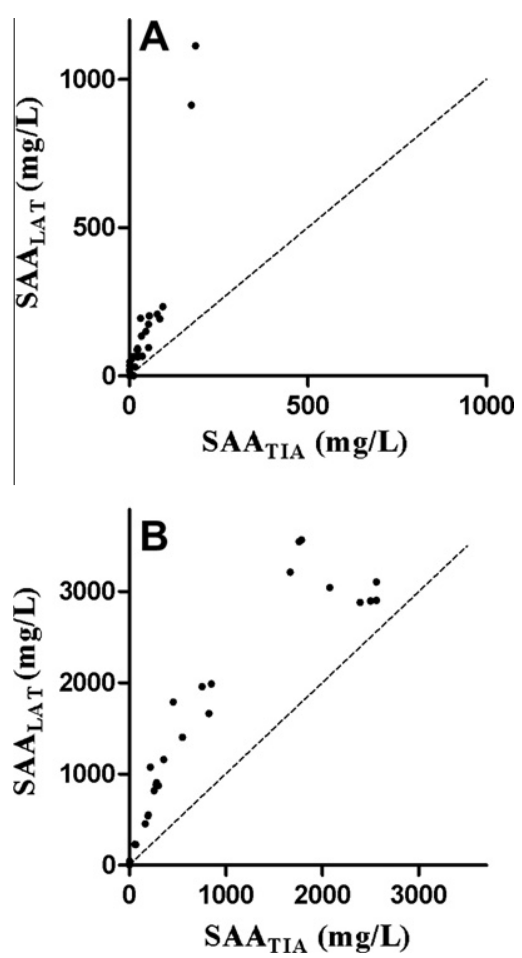

Fig. 2. Comparison of feline $(A)$ and equine $(B)$ serum amyloid $A(S A A)$ concentrations measured by the novel latex agglutination turbidimetric immunoassay (LAT) and a previously validated commercial turbidimetric immunoassay (TIA). The dashed line illustrates line of agreement $(\mathrm{Y}=\mathrm{X})$.

\section{Overlap performance}

As illustrated in Fig. 3, higher concentrations of SAA were observed in dogs with systemic inflammation compared to healthy dogs or diseased dogs without systemic inflammation, as expected for a major APP. High concentrations of SAA were observed in patients with systemic inflammation, and low concentrations were measured in patients without inflammation (Fig. 3). The multiple comparison test showed that this difference was significant $(P<0.05)$.

\section{Discussion}

In the present study the novel LAT based on monoclonal antihuman SAA antibodies was evaluated for measurements of SAA in dogs, cats, and horses. The reliability of the assay was found to be acceptable for determination of SAA in canine, feline, and equine serum with comparable inter- and intra-assay imprecision across the species. The observed inter- and intra-assay CVs were comparable with commercially available methods for measurement of feline and equine SAA and canine CRP, respectively

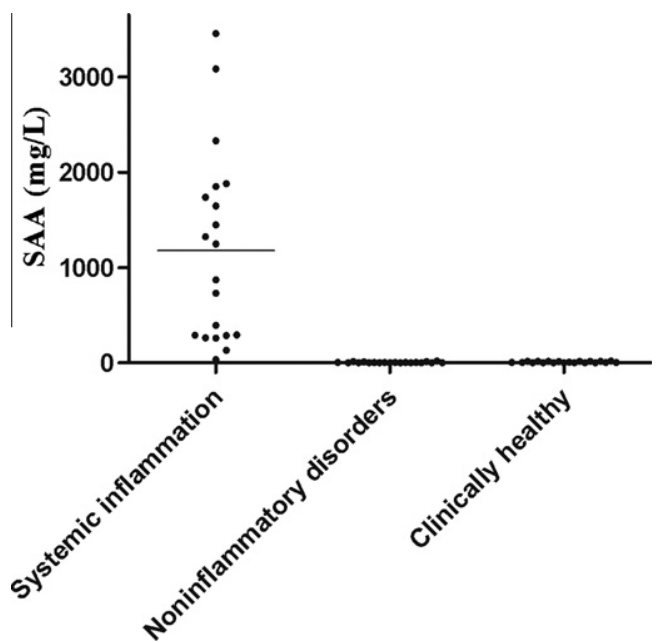

Fig. 3. Serum amyloid A (SAA) concentrations in serum from healthy and diseased dogs illustrating the overlap performance of SAA in the distinction of disorders with and without inflammation. Horizontal lines, mean concentrations.

(Kjelgaard-Hansen et al., 2003; Hansen et al., 2006; Jacobsen et al., 2006a). Acceptable linearity was observed within a clinically relevant working range.

Specifically for equine SAA, where extremely high SAA values are occasionally observed in clinical disease (Jacobsen et al., 2006b), no prozone effect was observed in concentrations up to $10,000 \mathrm{mg} / \mathrm{L}$. The assessed absolute concentration of $10,000 \mathrm{mg} / \mathrm{L}$ may be due to the heterologous calibration of the assay rather than the SAA concentrations actually being so high. However, the key finding is the lack of a prozone effect, meaning that no false low concentrations were measured, even when the LAT antibodies were completely saturated with SAA. A plateau was reached when concentrations exceeded $2500 \mathrm{mg} / \mathrm{L}$, and precise monitoring in patients with SAA concentrations persistently exceeding this limit will therefore need further dilution, either manually or as a second reflex dilution step. Because of the short half-life of SAA, the concentration is, however, expected to decrease quickly in diseased horses subdued to effective treatment (Jacobsen and Andersen, 2007). Horses with SAA concentrations persistently $>2500 \mathrm{mg} / \mathrm{L}$ are therefore likely to have a persistent acute phase reaction, making SAA concentrations persistently $>2500 \mathrm{mg} / \mathrm{L}$ clinically useful.

Acceptable linearity under dilution was observed in all species. Even though measurements of feline SAA significantly deviated from a linear model determined by runs test, slopes and Y-intercepts did not differ significantly from 1 and 0 , respectively, in any of the three species (Table 2). Therefore, the accuracy of the assay seems to be sufficient for clinical diagnostic purposes.

Wider ranges of concentrations of feline and equine SAA was measured in the present study $(0-1113 \mathrm{mg} / \mathrm{L}$ and $0->2500 \mathrm{mg} / \mathrm{L}$, respectively) compared to similar measurements in other studies where feline SAA ranged from 0 to $153.5 \mathrm{mg} / \mathrm{L}$ (Kajikawa et al., 1999; Sasaki et al., 2003; Hansen et al., 2006; Tamamoto et al., 2008, 2009) and equine SAA from 0 to $800 \mathrm{mg} / \mathrm{L}$ (Hultèn et al., 1999; Hultén and Demmers, 2002; Jacobsen et al., 2005, 2006b; Jacobsen and Andersen, 2007). Measurements of feline and equine SAA using the novel LAT systematically exceeded SAA measurements of the same samples when compared to a previously validated automated method used as reference method. The difference can most likely be explained by differences in the affinity of the antibodies used in the assays: the monoclonal antibodies in the novel LAT were selected to optimise cross-reactivity to companion animal SAA, whereas the antibodies of the previously validated assay were optimised for human use only. As both 
assays are calibrated with recombinant human SAA (thus measuring human SAA equivalents), a higher affinity to a specific SAA (e.g. feline SAA) will translate into a higher SAA concentration read from the calibration curve. If made available, the use of species specific calibration material would compensate for this. The disagreement should, however, not result in any problems of clinical significance, as long as the clinical decision levels for the novel LAT are adjusted for the increased affinity. The high degree of correlation between the two methods for measuring SAA ensures a high degree of agreement on clinical classification between the novel LAT and the previously validated method.

The overlap performance of SAA as a diagnostic marker of the acute phase response in cats and horses has been well documented, showing high concentrations of SAA in diseased animals with systemic inflammation and low SAA concentrations in animals with disorders without systemic inflammation or healthy (Hultèn et al., 1999; Kajikawa et al., 1999; Sasaki et al., 2003; Jacobsen and Andersen, 2007; Tamamoto et al., 2008). In the present study, the overlap performance of canine SAA measured by the LAT was analysed in order to investigate whether comparable patterns of concentrations were observed. SAA was measured in three groups of dogs, with a comparable spectrum of ages and breeds. Based on SAA measurements, dogs with marked acute phase responses could be distinguished from healthy dogs and diseased dogs without systemic inflammation as in other species (Jacobsen and Andersen, 2007; Tamamoto et al., 2008) and with other positive APPs (Kjelgaard-Hansen et al., 2003; Nakamura et al., 2008; Yuki et al., 2010).

This novel LAT can seemingly be used for diagnostic purposes in the assessment of the acute phase response in dogs by measuring SAA concentrations between 0 and $>3000 \mathrm{mg} / \mathrm{L}$. In most cases, a more narrow spectrum of SAA concentrations are measured in dogs (Dabrowski et al., 2009; Martinez-Subiela et al., 2011), but concentrations $>3000 \mathrm{mg} / \mathrm{L}$ are occasionally observed (Lowrie et al., 2009), making the working range of the assay relevant for routine use in a general clinical setting.

\section{Conclusions}

The LAT assay measures SAA in canine, feline, and equine serum with analytical and overlap performance acceptable for clinical purposes. The method is automated, rapid, and applicable for random-access analysers facilitating routine use in diagnostic laboratories. Furthermore, since the LAT is based on monoclonal antibodies, it may be associated with a strong long-term and inter-batch performance.

\section{Conflict of interest statement}

T. Ichiyanagi is an employee of EIKEN Chemical Company, Tokyo, Japan that manufactures the assay investigated in the present study. None of the other authors have any financial or personal relationships that could inappropriately influence or bias the content of the paper.

\section{Acknowledgements}

A special thank you to the employees at the Biochemical Research Laboratory, at EIKEN Chemical Company, Tokyo, Japan for their work in the development of the assay and to the staff at the Central Laboratory, Department of Small Animal Clinical Sciences, University of Copenhagen for technical support during the evaluation of the assay. Additional thanks to Heidi Gertz Andersen and Professor Peter Heegaard at the National Veterinary Institute,
Division of Veterinary Diagnostics and Research, the Technical University of Denmark for very important inputs.

\section{Appendix A. Supplementary material}

Supplementary data associated with this article can be found, in the online version, at http://dx.doi.org/10.1016/j.tvjl.2012.05.007.

\section{References}

Büttner, J., Borth, R., Boutwell, H., Broughton, P., 1980. International federation of clinical chemistry. Approved recommendation (1978) on quality control in clinical chemistry. Part 2. Assessment of analytical methods for routine use. Journal of Clinical Chemistry and Clinical Biochemistry 18, 78-88.

Cerón, J., Eckersall, P., Martinez-Subiela, S., 2005. Acute phase proteins in dogs and cats: Current knowledge and future perspectives. Veterinary Clinical Pathology 34, 85-99.

Chikamune, T., Katamoto, H., Nomura, K., Ohashi, F., 1998. Lipoprotein profile in canine pancreatitis induced with oleic acid. Journal of Veterinary Medical Science 60, 413-421.

Dabrowski, R., Wawron, W., Kostro, K., 2007. Changes in CRP, SAA and haptoglobin produced in response to ovariohysterectomy in healthy bitches and those with pyometra. Theriogenology 67, 321-327.

Dabrowski, R., Kostro, K., Lisiecka, U., Szczubial, M., Krakowski, L., 2009. Usefulness of C-reactive protein, serum amyloid A component and haptoglobin determinations in bitches with pyometra for monitoring early postovariohysterectomy complications. Theriogenology 72, 471-476.

Eckersall, P., 2004. The time is right for acute phase protein assays. The Veterinary Journal 168, 3-5.

Hagman, R., 2011. Serum $\alpha$-1-acid glycoprotein concentrations in 26 dogs with pyometra. Veterinary Clinical Pathology 40, 52-59.

Hansen, A., Schaap, M., Kjelgaard-Hansen, M., 2006. Evaluation of a commercially available human serum amyloid A (SAA) turbidometric immunoassay for determination of feline SAA concentration. Veterinary Research Communications 30, 863-872.

Hultén, C., Demmers, S., 2002. Serum amyloid A (SAA) as an aid in the management of infectious disease in the foal: Comparison with total leukocyte count, neutrophil count and fibrinogen. Equine Veterinary Journal 34, 693-698.

Hultèn, C., Talamo, R., Suominen, M., Burvall, K., Marhaug, G., Forsberg, M., 1999. A non-competitive chemiluminescence enzyme immunoassay for the equine acute phase protein serum amyloid A (SAA) - A clinically useful inflammatory marker in the horse. Veterinary Immunology and Immunopathology 68, 267281.

Jacobsen, S., Andersen, P., 2007. The acute phase protein serum amyloid A (SAA) as a marker of inflammation in horses. Equine Veterinary Education 19, 38-46.

Jacobsen, S., Jensen, J., Jensen, A., Thoefner, M., 2005. Use of serum amyloid A and other acute phase reactants to monitor inflammatory response after castration in horses: A field study. Equine Veterinary Journal 37, 552-556.

Jacobsen, S., Kjelgaard-Hansen, M., Petersen, H., Jensen, A., 2006a. Evaluation of a commercially available human serum amyloid A (SAA) turbidometric immunoassay for determination of equine SAA concentrations. The Veterinary Journal 172, 315-319.

Jacobsen, S., Thomsen, M., Nanni, S., 2006b. Concentrations of serum amyloid A in serum and synovial fluid from healthy horses and horses with joint disease. The American Journal of Veterinary Research 67, 1738-1742.

Jensen, A., Kjelgaard-Hansen, M., 2006. Method comparison in the clinical laboratory. Veterinary Clinical Pathology 35, 276-286.

Jensen, A., Bomholt, M., Moe, L., 2001. Preliminary evaluation of a particle enhanced turbidometric immunoassay (PETIA) for the determination of serum cystatin clike immunoreactivity in dogs. Veterinary Clinical Pathology 30, 86-90.

Kajikawa, T., Furuta, A., Onishi, T., Tajima, T., Sugii, S., 1999. Changes in concentrations of serum amyloid A protein, $\alpha_{1}$-acid glycoprotein, haptoglobin, and C-reactive protein in feline sera due to induced inflammation and surgery. Veterinary Immunology and Immunopathology 68, 91-98.

Kjelgaard-Hansen, M., 2010. Comments on measurements of C-reactive protein in dogs. Veterinary Clinical Pathology 39, 402-404.

Kjelgaard-Hansen, M., Jacobsen, S., 2011. Assay validation and diagnostic applications of major acute-phase protein testing in companion animals. Clinics in Laboratory Medicine 31, 51-70.

Kjelgaard-Hansen, M., Jensen, A., Kristensen, A., 2003. Evaluation of a commercially available human C-reactive protein (CRP) turbidimetric immunoassay for determination of canine serum CRP concentration. Veterinary Clinical Pathology 32, 81-87.

Klenner, S., Bauer, N., Moritz, A., 2010. Evaluation of three automated human immunoturbidimetric assays for the detection of C-reactive protein in dogs. Journal of Veterinary Diagnostic Investigation 22, 544-552.

Lowrie, M., Penderis, J., Eckersall, P., McLaughlin, M., Anderson, T., 2009. The role of acute phase proteins in diagnosis and management of steroid-responsive meningitis arteritis in dogs. The Veterinary Journal 182, 125-130.

Martinez-Subiela, S., Strauss-Ayali, D., Ceron, J., Baneth, G., 2011. Acute phase protein response in experimental canine leishmaniosis. Veterinary Parasitology 180, 197-202. 
Nakamura, M., Takahashi, M., Ohno, K., Koshino, A., Nakashima, K., Setoguchi, A., Fujino, Y., Tsujinoto, H., 2008. C-reactive protein concentration in dogs with various diseases. Clinical Pathology 70, 127-131.

Sasaki, K., Ma, Z., Khatlani, S., Okuda, M., Inokuma, H., Onishi, T., 2003. Evaluation of feline serum amyloid A (SAA) as an inflammatory marker. Journal of Veterinary Medical Science 65, 545-548.

Tamamoto, T., Ohno, K., Ohmi, A., Goto-Koshino, Y., Tsujimoto, H., 2008. Verification of measurement of the feline serum amyloid A (SAA) concentration by human
SAA turbidimetric immunoassay and its clinical application. Clinical Pathology 70, 1247-1252.

Tamamoto, T., Ohno, K., Ohmi, A., Seki, I., Tsujimoto, H., 2009. Time-course monitoring of serum amyloid $\mathrm{A}$ in a cat with pancreatitis. Veterinary Clinical Pathology 38, 83-86.

Yuki, M., Hiroshi, I., Takase, K., 2010. Serum $\alpha$-1-acid glycoprotein concentration in clinically healthy puppies and adult dogs and in dogs with various diseases. Veterinary Clinical Pathology 39, 65-71. 